\title{
L'INFLUENCE DU DROIT FRANÇAIS SUR LE DROIT BRÉSILIEN ${ }^{*}$
}

\author{
Antonio Junqueira de Azevedo \\ Professeur Titulaire du Département du Droit Civil \\ Ex-Directeur de la Faculté de Droit de 1'Université de São Paulo
}

Un exposé sur les influences du droit français sur le droit brésilien exige au préalable une explication historique. Nous diviserons donc notre conférence en deux parties: l'une, générique, qui prenda le droit brésilien à ses origines, encore lié au droit portugais et se prolongera après l'Indépendance, jusqu'à la proclamation de la République (1889); et l'autre, espécifique, à partir de la République, et qui sera relative aux différentes branches du Droit (droit civil, comercial, constitutionnel, administratif, pénal, etc.) avec des thèmes délimités. Au fond, cette division de l'exposé obéit à un double critère, ce qui peut ne pas être très logique mais nous paraît adapté à son objet: la première partie est chronologiquement antérieure et le droit y sera étudié quant à ses sources et à sa doctrine. La deuxième est plus récente et le Droit y sera étudié de manière fractionnée, dans ses différents domaines.

\section{PREMIĖRE PARTIE - RÉSUMÉ HISTORIQUE}

C'est justement au XVI siècle, dans la splendeur de la civilisation portugaise qu'a lieu la première grande influence du droit français sur le droit portugais. Le royaume de Portugal semble avoir été le premier à se consolider en Europe; dès le XII siècle, avec le roi Alphonse Henriquès, le Portugal avait approximativement son territoire actuale et une structure administrative propre. Il avait une langue nationale et avait été reconnu comme royaume indépendant aussi bien par le roi de Castille (Alphonse VII, en 1144) que par le Pape (Alexandre III, en 1179, par la Bulle Manisfestis Probatum). La précocité du Portugal comme Étatnation s'est révélée historiquement avantageuse. Il a pu se projeter dans le concert

- Conférence proferée à l'Université Paris II, le 26 novembre 1993. 
des nations, grâce aux hauts faits des XIV et XV siècles, avec les grandes navigations et les découvertes: îles Canaries (certainement encore au XIV), Madère et les Açores (occupées pendant la première moitié du XV), Cap Bojador (1434, par Gil Eanes), passage du Cap de Bonne Espérance (Le Cap, autrefois appelé des Tourmentes, 1487, par Bartolomeu Dias) et finalement l'arrivée en Inde avec Vasco de Gama, en 1498. Le 22 avril 1500, le Brésil est officiellement découvert par Pedro Álvares Cabral, sur le chemin des Indes. Au commencement du XVI siècle, le Portugal est donc à son apogée tant dans le domaine des arts que pour l'économie. Juridiquement aussi, il est assez précoce; en 1447, sous le règne d'Alphonse V (mais la régence de l'Infant Dom Pedro), est approuvée la réunion, en un seul corpus, de toutes les lois du royaume; ceci a été fait en cinq livres, qui on pris le nom d'Ordenações Afonsinas. Les livres I et II traitaient des règlements de l'organization judiciaire et administrative, des relations entre les organes du gouvernement et le clergé et des privilèges de la noblesse; le livre III de la procédure judiciaire, le livre IV du droit privé et le $\mathrm{V}$ du droit pénal. Peu après, le roi Manuel, justement appelé l'Heureux, a fait refaire et actualiser ce corpus de lois; ce qui a été réalisé exactement comme, plus tard, on fera les codes modernes c'est-à-dire de forme unitaire, sans références aux lois antérieures, en innovant, et avec une rédaction simplifiée, que nous povons considérer comme moderne. Ce sont les Ordenações Manuelinas, publiées définitivement en 1521. (Il y a eu une édition antérieure, de 1514, qui a été retirée, sous peine d'amende et de bannissement pour éviter toute confusion entre les deux textes). Les Ordenações Manuelinas ont la même distribution des matières que les Alfonsinas, en cinq livres: les deux premiers de droit public, le troisième, très complet, de droit processuel, le quatrième de droit privé et le cinquième de droit pénal.

La grande influence du droit français a été alors celle de l'humanisme juridique, de Budé, Cujas, Alciati, Doneau, Mudée et leur préoccupation d'apuration des textes du Corpus Juris, en les libérant de l'excès de commentaires, leur activité de critique historique établissant la signification originale des règles juridiques romaines, et leur zèle pour le style. Les lois portugaises étaient à cette époque très perfectionnées, spécialement en matière de droit public mais, quant au droit privé, le Livre Quatre des Ordenações Manuelinas, bien que clair, ne traitait que de questions spécifiques; au fond, la plupart des questions de droit privé continuaient à être résolues en se fondant sur le Corpus Juris Civilis, plus 
spécialement sur le Digeste. Il s'ensuit que l'enseignement du droit privé avait pour base le droit romain tel qu'il était présenté dans la glose d'Acursio et accompagné des commentaires des post-gloseurs, spécialement de Bartolo. Sous influence française, surgit alors le courant dit école cultivée qui réagit contre les modèles méthodologiques des commentateurs; en faisait partie Antonio de Gouveia (Antoine ou Antonius Gouveanus mort en 1556) qui a été étudiant à Paris en 1527 et ensuite professeur d'humanités à Bordeaux, Toulouse, Avignon, Lyon et Paris, et défendait "l'union de l'étude du droit et des lettres humanistes". et aussi Manuel da Costa, Aires Pinhel et Diogo de Sá.'

Le conservatisme des juristes portugais n'a cependant jamais permis que l'esprit critique de l'humanisme juridique français soit levé à ses dernières conséquences; on n'a pas admis, au Portugal, que les textes du Corpus Juris luimême puissent être mis en doute, par exemple à travers l'étude des interpolations. L'apologie de la libre recherche sur le droit (ou libere philosophari) n'a touché que la libération des textes romains des commentaires des gloseurs et postgloseurs; cela apparaît clairement dans la réforme de l'Université faite par Juan III, successeur de D. Manuel, dès 1539 (Réglement de l'Institut), où on déconseillait formellement la citation exhaustive d'opinions et où on prescrivait de chercher la solution des cas directement dans les textes légaux. ${ }^{2}$ De toute manière, donc, par l'influence française en matière de droit privé, les doctrinaires ont été remplacés par les lois. Au cours des siècles suivants, XVII et XVIII, au Portugal, les lois perdront, à leur tour, de leur importance face à la jurisprudence.

Mais il apparaît plus que nécessaire, à ce niveau de l'exposé, de laisser de côté tout ce qui a pu arriver au Portugal, et de passer à ne parler que de la situation du Droit au Brésil; et bien que ce ne soit pas à proprement parler l'influence du droit français sur le droit brésilien, il vaut la peine de rappeler que, dans le XVI lui-même, dès 1504, s'intensifiant sous les règnes de François $1^{\text {er }}$ (1515-1547) et Henri II (1547-1559), les Français, à travers des corsaires plus ou moins protégés par leur roi, ont été fréquemment sur les côtes brésiliennes. Il en est résulté deux conséquences importantes sur nos structures juridiques

1 . Voir Antonio Manoel Hespanha, Historia das Instituições, Coimbra, Livraria Almedina, 1982, p. 505; Nuno Espinosa Gomes da Silva, Historia do Direito Português, 2* ed., Lisboa, Fundação Calouste Gulbenkian; idem Humanismo e Direito em Portugal no século XVI, Lisboa, 1964.

2. Voir les oeuvres citées dans la note antérieure. 
administratives: la première, en 1534, quand le roi de Portugal, Jean III (15211558), pour mieux défendre la colonie, après plusieurs échanges de lettres et de traités avec le roi de France (Fontainebleau, 1531 et Bayonne, 1536) a établi le système des capitaineries héréditaires (au nombre de 15); et la seconde, quand Estácio de Sá, neveu du Gouverneur Général du Brésil, Mem de Sá, après l'exclusion des Français amenés par Villegagnon (Nicolas Durand de Villegagnon, qui était sous le patronnage de l'amiral Gaspard de Coligny) a ordonné la fondation, dans la Baie de Guanabara, de la ville de Rio de Janeiro (1565). ${ }^{3}$

L'arrivée des caravelles portugaises dans le territoire qui sera le Brésil, et la pose de bornes en pierre qui représentaient la prise de possession de la nouvelle terre ont eu la curieuse conséquence que les lois portugaises, pour ainsi dire au côté des marins, ont également débarqué sur les plages brésiliennes et ont ensuite étendu leur vigueur; à mesure que les explorateurs, surtout ceux qu'on appelle les Bandeirantes, avançaient dans les terres, connaissant de nouvelles rivières, occupant les forêts et les champs, les mêmes lois portugaises les accompagnaient, comme une ombre. Il y a dans cette matière, même si cela échappe à notre thème, des conséquences très intéressantes: dans ce qu'on appelle sesmarias par exemple, d'où sont issues les grandes fermes (latifundi) brésiliennes, le même texte légal portugais voit ses intentions totalement inversées; dans les Ordenações Manuelinas, il y avait une règle que nous dirions aujourd'hui hautement progressiste en ce sens que les terres non cultivées par leurs propriétaires, c'est-à-dire les terres en friche, devaient être distribuées par les autorités pour qu'on puisse les cultiver. L'exigence pour la distribution était la suivante: pouvoir profiter des terres distribuées. Mais cette règle, au Portugal, profitait aux agriculteurs non propriétaires, les sans-terre, contre les propriétaires négligents. Mais au Brésil, toutes les terres étaient incultes et pouvaient donc être distribuées. Il falait cependant, pour les recevoir, avoir les moyens de les cultiver. Et elles étaient si nombreuses qu'elles ne pouvaient être distribuées qu'à ceux qui avaient beaucoup de capital pour défricher de grandes zones couvertes de forêts et affronter les indiens; ceux qui prétendaient recevoir les sesmarias devaient prouver en avoir les moyens, c'est-à-dire avoir des esclaves, de l'argent, des instruments agricoles, des armes. D'où le fait que la même règle qui, au Portugal, avait une

3. Waldemar Ferreira, História do Direito Brasileiro, São Paulo, Max Limonad, 1955, t. 3, p. 301, Au XVII, les français s'etabliront de nouveau au Brésil, cette fois-ci au Maranhão. 
portée sociale, en est venue au Brésil à favoriser les riches et la formation des latifundi.

À partir de 1580 , le Portugal, déjà en décadence, a été uni à l'Espagne, sous les règnes de Phillipe II, III et IV. ${ }^{4}$ En 1649 a eu lieu la restauration de la monarchie lusitaniene et Jean IV, le premier de la dynastie des Bragances, a assumé le trône, mais, malgré cela, on ne peut pas dire que, au cours des XVII et XVIII siècles, ait été arrêté le processus de décadence de la civilisation portugaise. Au Brésil, au contraire, il y a eu développement de la population et progrès économique; au XVIII siècle, avec la découverte des mines d'or, le Brésil a beaucoup progressé et il est possible d' affirmer qu'à la fin du siècle, l'économie brésilienne a dépassé celle du Portugal. Cette situation, malheureusement, ne trouvait pas de correspondance du point de vue culturel, puisqu'il n'y avait au Brésil ni Université, ni école supérieure. ${ }^{5}$

La première grande influence culturelle française au Brésil date de cette époque, la fin du XVIII siècle. Bien qu'ayant juridiquement la nationatilé portugaise, les Brésiliens, en fait, se distinguaient dès cette époque des personnes originaires de la Métropole; ils avaient aussi, à des degrés plus ou moins grands, du sang indien ou noir; ils étaient en conséquence en général plus bruns quand ils n'étaient pas métis affirmés. De plus, ils parlaient un portugais plus lent, avec un vocabulaire qui n'était pas toujours identique. Ils voyaient le gouvernement comme quelque chose qui leur était étranger, parce que la vie quotidienne au Brésil était très autonome; à cause de la géographie et de l'extension du territoire, le gouvernement avait peu de participation dans les activités courantes de la population. Les idées de Jean-Jacques Rousseau et des Encyclopédistes ont été en vogue chez le petit nombre des intellectuels brésiliens, avant même la Révolution Française. En 1789, le gouvernement portugais a fait arrêter ceux qu'on a appelé les Inconfidents de Minas Gerais, qui voulaient l'indépendance du Brésil. Leur représentant le plus célèbre, Tiradentes, a été rendu et est devenu le héros national

4. Sous le règne des Phillipes, Portugal a conservé son autonomie administrative. Les Ordenações Manuelinas ont été remplacées par les Ordenações Filipinas (1604) qui étaient l'oeuvre de Portugais et non d'Espagnols.

5. Il y avait alors dans la structure juridique portugaise un tribunal supérieur, à Lisbonne, appelé Casa da Suplicação, équivalent au Tribunal de Cassation; sous lui, cinq Tribunaux de seconde instance à Porto, Evora, Goa (Inde), Rio de Janeiro et Bahia (Brésil) trois donc hors du Portugal dont deux au Brésil. 
du Brésil (en réalité, il n'était pas à proprement parler un des chefs du mouvement, puisqu'il s'agissait d'une personne simple, un sous lieutenant, mais il s'est révélé réellement décidé à l'indépendance et il l'a proclamé pendant son procès, étant le seul à être exécuté). Il est intéressant d'observer qu'à partir de ce moment et pendant environ deux cents ans, la culture française a été la principale source étrangère de notre formation intelectuelle (et c'est seulement dans le siècle actuel, aux environs de la décade de 60 , que ce rôle a été assumé par la culture américaine, aujourd'hui largement prédominante). Même le peu que les Inconfidentes connaissaient de la récente Constitution américaine, c'était à travers une traduction française. Souvent les auteurs suivis n'étaient pas ceux de la première ligne; par exemple, comme on peut le voir dans les minutes de l'instruction, à travers les registres du séquestre des biens des révoltés, les oeuvres de l'Abbé Reynal étaient très lues.

L'établissement de l'Empire napoléonien, peu après, a eu des conséquences directes sur le "statut" juridique du Brésil; on sait que l'Empereur des Français, à cause du Blocus Continental, a envoyé le général Junot, en 1807, envahir le Portugal et déposer la Maison de Bragance. À cette occasion, la Cour portugaise a mis à exécution un plan qui avait été conçu dans un autre cas, quand la menace venait de l'Espagne. Pratiquement toute l'élite portugaise, près de 10.000 personnes, dont la reine Maria $1^{\text {ère }}$, la folle, le prince régent, le futur Jean VI, le haut clergé, les ministres, les juges des tribunaux supérieurs, les nobles, les riches commerçants, les cadres supérieurs de l'administration et même les domestiques du Palais sont partis pour le Brésil, s'établissant à Rio de Janeiro. Il s'agit d'un cas unique dans l'histoire. A partir de là, Rio de Janeiro, ville américaine, est devenue la capitale d'un royaume européen; même après la chute de Napoléon et la libération du Portugal, la situation a continué, durant jusqu'en 1821. De plus, après l'Indépendance du Brésil, en 1822, et pendant plus de 67 ans, c'est-à-dire jusqu'à la proclamation de la République, une famille dynastique européenne a régné sur des terres du Nouveau Monde.

Une autre conséquence a été que, précisément en 1808, les Brésiliens ont occupé une partie du territoire français. Je veux dire que nous avons pris la Guyane française, l'incorporant à notre territoire. Ce qui a fait que la France, en accord avec 1 Espagne, ait exigé au Congrès de Vienne, en 1815, qu'un représentant brésilien, en plus du représentant du Portugal, signe le Traité de Vienne. Les 
gouvernements européens se mettaient ainsi à l'abri de toute proclamation d'indépendance du Brésil, dont ils reconnaissaient, de facto, la situation d'autonomie. Et, en conséquence le roi de Portugal a élevé le Brésil à la catégorie de royaume, le désignant par Décret-loi du 16 décembre 1815 royaume uni à celui du Portugal. Mais cette date n'est pas considérée au Brésil comme celle de l'Indépendance, parce qu'il n'y a eu que peu de changements, la Cour continuant à Rio de Janeiro. L'Indépendance brésilienne viendra seulement quelques années plus tard, en 1822, quand les Portugais exigèrent le retour du roi à Lisbonne; d'ailleurs, elle est le résultat d'un accord entre des nationalistes brésiliens conservateurs et le prince héritier portugais, Pierre $1^{\text {er }}$ Le Brésil s'est séparé du Portugal mais la Maison régnante a continué jusqu'à la proclamation de la République, en 1889.

À partir de 1804, comme on le sait, l'influence du Code Civil français est presque universelle. Au Brésil, les lois portugaises ont continué à être en vigueur après l'Indépendance. La Constitution octroyée en 1824 prévoyait l'élaboration de codes civil et pénal (art. 179, n. 18). En 1827, ont été installés les cours de droit de São Paulo et d'Olinda. Cependant, bien que les professeurs aient beaucoup lu les auteurs français, et malgré la détermination constitutionnelle, nous avons eu beaucoup de difficultés pour élaborer le Code Civil; les raisons n'en étaient pas le manque de juristes pour le faire puisque, en réalité, plusieurs projets ont été élaborés pendant toute la période impériale brésilienne, spécialement la fameuse "Ebauche" de Teixeira de Freitas qui a servi de base pour le Code Civil argentin (résultat du projet de Valez-Sarsfield, qui a déclaré s'être basé sur les travaux de Teixeira de Freitas). Les raisons, à mon avis, sont que le grand juriste cité, chargé du premier projet, n'était pas d'accord avec la distribution des matières dans le Code Civil français; auparavant, en 1858, il avait déjà élaboré une "Consolidation des Lois Civiles" avec une partie générale comme en aurait par la suite le BGB, et puis il a élaboré un projet, "l'Ebauche" réellement innovateur, précurseur des temps futurs, qui était basé sur la distinction entre les droits réels et les droits personnels ou obligations et unifiait droit civil et droit commercial. Ce projet a été refusé par le Ministre José de Alencar, personnalité conservatrice (à l'instar des intellectuels romantiques français de son temps, Chateaubriand, Lamartine ou Victor Hugo, il a été aussi un grand romancier). Une autre raison des difficultés que connaissait le Code Civil, qui a continué pour les projets postérieurs, était typiquement brésilienne: l'esclavage. La production agricole (canne à sucre, 
café, etc.), les travaux domestiques et l'ampleur du capital employé dans la traite des esclaves, empéchaient son abolition mais, en même temps, les juristes brésiliens ne voulaient pas élaborer un Code Civil qui paraisse aux yeux du Monde consacrer cette iniquité. La conséquence en a été que, pendant tout l'Empire, nous n'avons pas eu de Code Civil (l'abolition date de 1888 et, en 1889, la République a été proclamée).

Pour leur part, le Code Pénal (1830), le Code de Procédure Pénal et le Code Commercial (1850) n'ont pas souffert d'influence directe de leurs congénères français; ils ont plutôt été basés sur la législation portugaise.

Nous pouvons synthétiser tout ce qui a été dit dans cette première partie en soulignant que:

$\left.1^{\circ}\right)$ la France, que ce soit directement, par des actes concrets, ou indirectement à travers ses intellectuels, a exercé une énorme influence sur le droit brésilien, c'est-à-dire sur le droit comme loi et les doctrines juridiques; elle a altéré les structures administratives, entrainant même un changement du statut juridique de la colonie, elle a fait bouger des intellectuels et formé des professeurs.

$2^{\circ}$ ) le droit français n'a cependant pas eu d'influence notable jusqu'à l'établissement de la République: aucun de nos codes, ni la législation civile n'ont été copiés ou adaptés, à cette époque, de la législation française.

\section{SECONDE PARTIE - LE DROIT ACTUEL}

Le droit brésilien postérieur à la proclamation de la République, soit pratiquement celui du $\mathrm{XX}$ siècle, présente la caractéristique suivante: selon la branche du Droit, il y a variation du pays qui exerce l'influence. Dans le droit processuel et le droit pénal, c'est l'Italie qui prédomine; dans le droit administratif et le droit international, c'est la France; dans le droit commercial, les États Unis; dans le droit civil, France, Allemagne et Portugal se disputent la primauté; dans le droit constitutionnel enfin, domaine qui préoccupe actuellement le juriste brésilien, les solutions françaises sont souvent citées, spécialement pour ce qui est de la forme de gouvernement, mais comme il y a la beaucoup de problèmes, on consulte également les Constitutions des autres pays. Il est possible de dire, paradoxalement, que l'originalité du droit brésilien actuel est de savoir copier. 
Pour ne pas nous étendre exagérément, voyons seulement l'influence française qui, comme il a été dit, est très forte dans le droit administratif, le droit international et dans notre spécialité, le droit civil, pour quelques uns de ses thèmes.

Le droit administratif brésilien, qui est enseigné dans nos cours juridiques depuis 1854, est entièrement tributaire du droit français. Il est vrai que nous n'avons pas de tribunaux administratifs décidant en dernière instance, comme le "Conseil d'État" Il existe une détermination constitutionnelle qui dit que "la loi n'exclura pas du Pouvoir Judiciaire une lésion on une menace au droit" (Constitution de la République, art. 5, XXXV), et un tribunal administratif, pour nous, ne fait pas partie du Pouvoir Judiciaire. Cependant, toute la discussion sur le service public entre ce qu'on appellait "l'école du Service Public" ou de Bordeaux qui a eu pour chef de file Léon Duguit, et le courant opposé de Maurice Hauriou, a eu une grande répercussion au Brésil. Sont aussi souvent cités les noms de Gaston Jèze, Henri Barthélémy, Roger Bonnard et d'autres plus récents Laubadère, Jean Valine, George Vedel, pour ne pas citer les noms d'aujourd'hui et la Revue Française de Droit Administratif.

Dans le droit international, qu'il soit public ou privé, l'influence des auteurs français sur les brésiliens est totale; outre les décisions de la Cour de La Haye et les traités internationaux, même certains écrivains allemands sont connus à travers des versions françaises. Henri Batifol a été, pendant des années, la référance constante. Par convenance, nous omettons les noms les plus récents.

Dans le droit civil, on peut dire que, à partir de l'entrée en vigueur du Code Civil, le $1^{\text {er }}$ janvier 1917, le droit brésilien a pris un nouveau cours. Du droit français, il a hérité la dichotomie: Code Civil séparé du Code Commercial; on n'a pas suivi l'idée d'unification de Teixeira de Freitas (concretisée en Italie, en 1942). Mais le Code Civil brésilien se détache complètement de la systématique du Code Napoléon; il a la division du "Burgerlich Gesetzbuch" de 1896, avec une Partie Générale et une Partie Spéciale. La Partie Générale suit exactement les leçons de la Pandectiste; elle a un Livre pour les personnes, un autre pour les biens et un troisième pour l'acte juridique (terminologie française mais, au fond, c'est le Rechtsgeschaft des allemands). La Partie Spéciale a quatre livres: Droit de la Famille, Droits Réels, Droit des Obligations et Droit des Sucessions (exactement comme le Code Civil allemand). 
Sur l'acte juridique, nous suivons la théorie pandectiste du Rechtsgeschaft et la théorie anti-causaliste de Planiol, mais la théorie des nullités des actes juridiques dans le droit brésilien accompagne la doctrine française au point que René David conseille à qui prétend l'étudier de lire l'oeuvre de l'écrivain français Solon, écrite en 1835 , et publiée en traduction brésilienne das une revue specialisée.

La conception de l'erreur comme défaut de l'acte juridique (art. $96 \mathrm{du}$ Code brésilien) est celle de l'art. 1169 du Code français et les civilistes suivent leurs collègues français en tout ce qui se réfère aux vices du consentement.

Le droit brésilien se sépare radicalement du droit français mais aussi du droit allemand pour ce qui est du système d'acquisition de la propriété immobilière. Pour le Code Napoléon, comme on sait, le contrat a pour effet de transmettre la proprieté. Dans le droit brésilien, la propriété ne s'acquiert pas solo consensu; il n'a pas adopté intégralement la systématique germanique, centrée sur un "accord formel de transmission" qui s'érige en "convention qui a effet réel" l'inscription sur le registre immobilier assumant le caractère d'acte juridique abstrait, c'est-à-dire, cet acte enregistré dans les livres fonciers, selon le BGB, se détache de l'acte juridique sous-jacent. Ainsi, au contraire du droit français où l'acte translatif produit tous les effets entre les parties, dans le droit germanique, c'est l'enregistrement de l'accord de transmission qui produit un effet probatoire absolu, d'une présomption juris et de jure. Pour le Code Civil brésilien, à la différence du Code Napoléon et du B.G.B., la transmission de la propriété immobilière ne se fait pas solo consensu mais il n'y a pas, non plus, d'accord de transmission séparé du contrat; on est plus fidèle à la conception du droit romain, pour lequel la propriété des choses ne se transfert pas par le contrat ("traditionibus et usucapionibus non nudis pactis, dominia rerum transferuntur") exigeant un acte matériel et concret qui, pour les biens meubles est la tradition, et pour les biens immobiliers, chez nous, l'inscription du contrat au Registre. Au Brésil comme dans l'ancienne Rome, il faut, pour l'acquisition de biens, comme disaient les juristes au Moyen-Age, titulus (le contrat) plus modus (la tradition pour les meubles et le registre du contrat pour les immeubles (voir sur l'acquisition des biens immobiliers dans le droit français, Paul Violet, Histoire du droit civil français, 3 ième ed., p. 665; Mazeaud, Leçons de Droit Civil, v. 2, n. 1.612; Planiol, Ripert et Boulanger, Traité Elémentare, v. 1, n. 2.885; Aubry et Rau, Cours, v. 2 paragraphe 207; Marty et 
Raynaud, Droit Civil, v. 2, n. 53; sur l'acquisition de la propriété immobilière dans le droit allemand, Ennecceurs, Kipp y Wolff, Tratado, Derecho de Cosas, v. 1, paragraphe 26 et suiv; Hedemann, Derechos Reales, paragraphe 9; sur l'acquisition de la propriété immobilière dans le droit brésilien, Soriano Neto, Publicidade Material e Registro Imobiliário, n. 59; Serpa Lopes, Curso de Direito Civil, v. 6, n. 338; Clóvis Bevilaqua, Direito das Coisas, v. 1, p. 147; Caio Mario da Silva Pereira, Instituições do Direito Civil, v. 4, n. 303; Orlando Gomes, Direitos Reais, cap. XIII et aussi, notre conférénce à l'Université de Rome, La Sapienza, le 5 février 1992).

On sent cepandant dans d'autres domaines civilistes la présence du droit français. La théorie de la responsabilité civile dans le Code Civil brésilien est totalement dérivée du Code Napoléon, dont l'article 1382 dit: "Tout fait quelconque de l'homme, qui cause à autrui un dommage, oblige celui par la faute duquel il est arrivé, à le réparer" Et l'article 1383 établit: "Chacun est responsable du dommage qu'il a causé non seulement par son fait, mais encore par sa négligence ou par son imprudence" L'art. 159 du Code Civil brésilien dispose: "Celui qui par action ou par omission volontaire, négligence ou imprudence viole le droit ou cause préjudice à autrui est obligé de réparer le dommage - La vérification de la culpabilité ou de la responsabilité sont réglées par ce que dispose ce Code" La théorie brésilienne de la responsabilité part de ce point et son développement sur la responsabilité par le fait de tiers ou par le fait des choses accompagne le droit français. Les décisions des juges français sont toujours citées en droit brésilien.

Il est également important d'observer que l'évolution de la théorie de la responsabilité dans le droit français, à partir de Josserand (De la Responsabilité des Choses Inanimées, Paris, 1897; Cours de Droit Civil Positif Français, v. 2, n. 486) et Gaston Morin (La Révolte du Droit contre le Code, page 62) amène les professeurs brésiliens à la doctrine de la responsabilité objective qui trouve chez nous des défenseurs importants (Alvino Lima; José de Aguiar Dias; Wilson Melo da Silva et les divers Projets d'un nouveau Code Civil). Aujourd'hui, dans cette matière on cherche aussi, des solutions en droit français pour les problèmes du droit des consommateurs malgré l'existence d'un Code de Defense du Consommateur très moderne. 
Dans le droit de la famille, le principe de l'irrévocabilité du régime des biens dans le mariage est présent dans les articles 230 du Code brésilien et 1396 du Code français. Bien qu'adoptant la doctrine romaine de la filiation légitime "pater is est quem nuptias demonstrant"- on trouve dans les deux codes, le français (art. 312) et le brésilien (art. 340) la même exception si intervient l'impossibilité physique de la cohabitation. Mas il faut dire que, maintenant, tout le droit de la famille est bouleversé an Brésil; la nouvelle Constitution (1988) a donné des principes en contradicton avec le Code Civil et on a beaucoup de problèmes de compatibilisation.

De nombreux autres points pouvaient être indiqués au Code Civil brésilien copiés du Code Civil des Français, rappelant à titre d'échantillon l'institution de la répétition de l'indu, le concept des dommages et intérêts, et les formes testamentaires, mais ce serait trop fatiguer les auditeurs. Nous croyons malgré ça, avoir donné une idée de ce vaste sujet et nous finissons à ce point. 